\title{
Cultivo de genótipos de palma forrageira sob agricultura biossalina como alternativa para incremento do aporte forrageiro do semiárido brasileiro: Uma revisão
}

\author{
Cultivation of forage cactus genotypes under biosalin agriculture as an alternative to increase \\ forage input from the Brazilian semiarid region: A review \\ Cultivo de genotipos de palmeras forrajeras bajo agricultura de biosalina como alternativa para \\ incrementar el aporte forrajero de la región semiárida brasileña: Una revisión
}

\author{
José Orlando Nunes da Silva \\ ORCID: https://orcid.org/0000-0001-7622-5095 \\ Universidade Federal Rural de Pernambuco, Brasil \\ E-mail: joseorlando.agro@gmail.com \\ George do Nascimento Araújo Júnior \\ ORCID: https://orcid.org/0000-0001-9284-4160 \\ Universidade Federal Rural de Pernambuco, Brasil \\ E-mail: georgearaujo.agro@gmail.com \\ Alexandre Maniçoba da Rosa Ferraz Jardim \\ ORCID: https://orcid.org/0000-0001-7094-3635 \\ Universidade Federal Rural de Pernambuco, Brasil \\ E-mail: alexandremrfj@gmail.com \\ Cleber Pereira Alves \\ ORCID: https://orcid.org/0000-0002-8796-6945 \\ Universidade Federal Rural de Pernambuco, Brasil \\ E-mail: cleberp.agro@gmail.com \\ Antonio Gebson Pinheiro \\ ORCID: https://orcid.org/0000-0002-5912-6906 \\ Universidade Federal Rural de Pernambuco, Brasil \\ E-mail: gebson10@hotmail.com \\ João Pedro Alves de Souza Santos \\ ORCID: https://orcid.org/0000-0003-0804-115X \\ Universidade Federal Rural de Pernambuco, Brasil \\ E-mail: peualves02@gmail.com \\ Luciana Sandra Bastos de Souza \\ ORCID: https://orcid.org/0000-0001-8870-0295 \\ Universidade Federal Rural de Pernambuco, Brasil \\ E-mail: sanddrabastos@yahoo.com.br \\ Thieres George Freire da Silva \\ ORCID: https://orcid.org/0000-0002-8355-4935 \\ Universidade Federal Rural de Pernambuco, Brasil \\ E-mail: thigeoprofissional@hotmail.com.br
}

\begin{abstract}
Resumo
No Semiárido brasileiro (SAB), a pecuária extensiva é predominante, onde alimentação dos animais é dependente da vegetação nativa, que devido a variabilidade climática não supre a necessidade dos rebanhos. Para contornar esse problema, a seleção de espécies de plantas forrageiras adaptadas ao clima Semiárido pode reduzir os impactos promovidos pelas adversidades climáticas sobre a pecuária. Nesse contexto, uma cultura que merece destaque é a palma forrageira, que devido ao seu metabolismo e características morfológicas, apresenta elevada produção de biomassa, sobretudo, em ambientes com elevadas temperaturas e déficit hídrico. De forma a incrementar a produtividade dessa cultura, face a variabilidade climática e as condições dos recursos hídricos existentes no $\mathrm{SAB}$, a adoção de práticas de resiliência agrícola, como a agricultura biossalina, pode ser uma alternativa viável, proporcionando sustentabilidade ao sistema. Nesta revisão, objetivou-se fornecer uma visão geral da capacidade de produção de diferentes clones de palma forrageira cultivadas em ambiente semiárido sob agricultura biossalina, como forma de incrementar o aporte de forragem aos sistemas de produção dessa cactácea. Essa revisão baseou-se em periódicos disponíveis em plataformas digitais como ScienceDirect, Scorpus, SciELO e Google Scholar, priorizando artigos publicados nos últimos 10 anos. Concluiu-se que as mudanças climáticas devem afetar a pecuária e que
\end{abstract}


sistema de produção com agricultura biossalina, associado aos melhores genótipos de palma forrageira é uma alternativa promissora para a produção de forragem no SAB.

Palavras-chave: Genótipos; Nopalea; Opuntia; Semiárido.

\begin{abstract}
In the Brazilian Semiarid Region (BSR), extensive livestock is predominant, where animal feeding is dependent on native vegetation, which due to climatic variability does not meet the needs of herds. To circumvent this problem, the selection of species of forage plants adapted to the Semiarid climate can reduce the impacts caused by climatic adversities on livestock. In this context, a crop that deserves to be highlighted is the forage cactus, which due to its metabolism and morphological characteristics, presents high biomass production, especially in environments with high temperatures and water deficit. To increase the productivity of this crop, given the climatic variability and the conditions of the water resources existing in the BRS, the adoption of agricultural resilience practices, such as biosalin agriculture, can be a viable alternative, providing sustainability to the system. This review aimed to provide an overview of the production capacity of different cactus forage clones grown in a semiarid environment under biosalin agriculture, as a way of increasing the amount of forage to the production systems of this cactus. This review was based on journals available on digital platforms such as ScienceDirect, Scopus, SciELO and Google Scholar, prioritizing articles published in the last 10 years. It was concluded that climate change should affect livestock and that the production system with biosalin agriculture, associated with the best genotypes of forage cactus is a promising alternative for the production of forage in the BRS.
\end{abstract}

Keywords: Genotypes; Nopalea; Opuntia; Semiarid.

\title{
Resumen
}

En el Semiárido Brasileño (SAB) predomina la ganadería extensiva, donde la alimentación animal depende de la vegetación nativa, que debido a la variabilidad climática no satisface las necesidades de los rebaños. Para sortear este problema, la selección de especies de plantas forrajeras adaptadas al clima Semiárido puede reducir los impactos provocados por las adversidades climáticas sobre el ganado. En este contexto, un cultivo que merece ser destacado es la palma forrajera, que por su metabolismo y características morfológicas, presenta alta producción de biomasa, especialmente en ambientes con altas temperaturas y déficit hídrico. Para incrementar la productividad de este cultivo, dada la variabilidad climática y las condiciones de los recursos hídricos existentes en el SAB, la adopción de prácticas de resiliencia agrícola, como la agricultura de biosalina, puede ser una alternativa viable, brindando sustentabilidad al sistema. El objetivo de esta revisión fue brindar un panorama de la capacidad de producción de diferentes clones de palma forrajera cultivados en un ambiente semiárido bajo agricultura de biosalina, como una forma de incrementar la cantidad de forraje a los sistemas de producción de este cactus. Esta revisión se basó en revistas disponibles en plataformas digitales como ScienceDirect, Scorpus, SciELO y Google Scholar, priorizando artículos publicados en los últimos 10 años. Se concluyó que el cambio climático debe afectar a la ganadería y que un sistema de producción con agricultura de biosalina, asociado a los mejores genotipos de palma forrajera, es una alternativa prometedora para la producción de forrajes en el SAB.

Palabras clave: Genotipos; Nopalea; Opuntia; Semi árido.

\section{Introdução}

O modelo de agricultura praticado na região semiárida brasileira é bastante dependente das condições climáticas que, devido as suas oscilações, promovem vulnerabilidade aos sistemas de produção agropecuária, resultando em perdas econômicas significativas (Moraes et al., 2019). As regiões semiáridas são caracterizadas por apresentarem elevada variabilidade espaço-temporal da precipitação pluvial, com média anual inferior a $800 \mathrm{~mm}$, demanda evaporativa superior a $2000 \mathrm{~mm} \mathrm{ano}^{-1}$, índice de aridez de Thornthwaite $\leq 0,50$, déficit hídrico anual $\geq 60 \%$, com ocorrência de secas periódicas, o que resulta em déficit hídrico na maior parte do ano (Medeiros et al., 2020; Sudene, 2017).

No semiárido brasileiro (SAB), uma das principais atividades econômica é a pecuária. Em geral, a alimentação dos animais é proveniente da vegetação nativa, i.e., Caatinga, a qual apresenta baixo conteúdo de matéria seca em sua biomassa $\left(<5 \mathrm{t} \mathrm{ha}^{-1}\right.$ ano $\left.^{-1}\right)$ e baixo suporte forrageiro $\left(<1 \mathrm{t} \mathrm{ano}^{-1}\right)$ (Dubeux et al., 2015; Queiroz et al., 2020). Logo, práticas mitigatórias e de resiliência agrícola, como a utilização de plantas forrageiras adaptadas ao clima da região, tornam-se uma alternativa para garantir alimento para os rebanhos, principalmente durante a época seca. Nesse contexto, uma cultura que merece destaque é a palma forrageira (Opuntia spp. e Nopalea spp.), que devido a suas características morfológicas, fisiológicas e anatômicas, apresenta boa adaptação à ambientes com altas temperaturas e déficit hídrico, exibindo ainda, elevada eficiência no uso da 
água (Nunes et al., 2020; Queiroz et al., 2015; Silva et al., 2015; Taiz; Zeiger, 2017). Além disso, essa cultura apresenta boa aceitabilidade pelos animais, alta digestibilidade, grande quantidade de carboidratos solúveis, baixo custo de produção e alto rendimento de biomassa (Cardoso et al., 2019; Moura et al., 2020; Santos et al., 2020).

Mesmo apresentando tais características de adaptação, estudos têm mostrado que a prática de irrigação no cultivo da palma forrageira em ambientes Semiárido, favorece o crescimento da planta, a emissão de cladódios, o índice de área dos cladódios e a redução no ciclo da cultura, contribuindo para o incremento na produtividade e oferta de forragem (Pereira et al., 2015; Queiroz et al., 2015; Rocha et al., 2017; Silva et al., 2020). Sob condições irrigadas, os cultivos de palma forrageira podem alcançar produtividades 1,26 vezes superiores aos cultivos em sequeiro, e quando comparada às pastagens nativas de ambientes semiárido, o rendimento de forragem dessa cactácea pode ser cerca de 50 a 60 vezes maior por unidade de área cultivada (Dubeux et al., 2015; Neupane et al., 2021). Vale salientar que as repostas produtivas dos clones de palma forrageira sob irrigação, variam conforme a espécie utilizada, i.e., Opuntia spp. e Nopalea spp. (Amorim et al., 2015; Barbosa et al., 2018; Silva et al., 2015; Rocha; Voltolini e Gava, 2017).

A irrigação utilizada de forma complementar à precipitação pluvial, torna o cultivo de palma forrageira mais estável ao longo do ciclo, uma vez que não é atingido pelos eventos de irregularidade climática, e.g., estiagem prolongada, (Santos; Donato; Cotrim Junior, 2020). Contundo, como na maioria das regiões de clima semiárido, no SAB os recursos hídricos presentes não apresentam boa qualidade, devido a presença de grande quantidade de sais (Masters et al., 2007; Pimentel \& Sharqawy, 2020), o que torna necessário o manejo adequado da água de irrigação. Nesse sentindo, a agricultura biossalina surge como uma alternativa sustentável (Khorsandi et al., 2020). Essa prática consiste no cultivo de plantas tolerantes utilizando águas com elevados níveis de sais, em ambientes que já sofrem com problemas de salinidade (Khorsandi et al., 2020). Dessa forma, os recursos hídricos de boa qualidade são preservados e poderão ser utilizados para outras finalidades, assim como, o desenvolvimento sustentável dessas áreas é beneficiado.

Visto que a tolerância da palma forrageira à salinidade pode variar conforme as espécies utilizadas, e a adoção da agricultura biossalina pode ser uma prática viável para remediar os problemas existentes com os recursos hídricos do Semiárido brasileiro, nesta revisão, objetivou-se fornecer uma visão geral da capacidade de produção de diferentes clones de palma forrageira cultivadas em ambiente semiárido sob agricultura biossalina, como forma de incrementar o aporte de forragem aos sistemas de produção dessa cactácea nessa região.

\section{Metodologia}

O presente estudo, trata-se de uma revisão de literatura de caráter qualitativo e informativo, baseada em periódicos disponíveis em plataformas digitais como ScienceDirect, Scorpus, SciELO e Google Scholar, nos idiomas português e inglês, tendo como base a metodologia utilizada por Pereira et al. (2018). Para facilitar a busca dos trabalhos científicos foram utilizadas palavras chaves como "palma forrageira", "clones", "genótipos", "semiárido", "Opuntia", "Nopalea", "cactácea", "água salina”, "salinidade", "mudança climática", "forragem" e "déficit hídrico", em ambos os idiomas. Priorizou-se os artigos publicados nos últimos 10 anos. 


\section{Revisão de Literatura}

\subsection{Crescimento populacional versus produção agropecuária e mudanças climáticas}

A população mundial deve crescer cerca de dois bilhões de habitantes nos próximos 30 anos, chegando a 9,7 bilhões até 2050, conforme dados da Organização das Nações Unidas - ONU (Theurl et al., 2020). Para atender a demanda de consumo por alimento dessa população, deve-se aumentar a produção em 60\% (FAO, 2016). No entanto, o processo de mudanças climáticas tem reduzido a disponibilidade hídrica e promovido alterações na fisiologia vegetal, o que gera a necessidade de aprimorar os cultivos a mitigarem as condições adversas do clima, e produzir mais por unidade de área, evitando o avanço do desmatamento (Vieira Filho \& Fishlow, 2017).

$\mathrm{O}$ aquecimento global, promovido pelo aumento dos gases do efeito estufa (GEE), i.e., dióxido de carbono $\left(\mathrm{CO}_{2}\right)$; metano $\left(\mathrm{CH}_{4}\right)$ e óxido nitroso $\left(\mathrm{N}_{2} \mathrm{O}\right)$, representa uma série de riscos à produtividade das culturas em todo o mundo (Leisner, 2020; Palit et al., 2020; Taiz \& Zeiger, 2017). Em estudo, pesquisadores apontaram reduções no rendimento médio dos vegetais em decorrência das mudanças climáticas, variando em $22 \%$ para um aumento de $250 \mathrm{ppm}$ de $\mathrm{CO}_{2}, 8,9 \%$ para um aumento em $25 \%$ de $\mathrm{O}_{3}$ e $31,5 \%$ caso a temperatura aumente em $4{ }^{\circ} \mathrm{C}$ (Scheelbeek et al., 2018). Além de promover redução nos teores nutricionais das culturas, devido a falhas na concentração de minerais e proteínas nos grãos (Loladze, 2014; Mcgrath \& Lobell, 2013; Myers, 2014).

Somado a isso, espera-se que ocorra um aumento na incidência de eventos climáticos extremos, como as grandes secas (Chen et al., 2015; Marengo, 2008). Até o final do século, os eventos de estiagem prolongadas tendem a ser mais intensos, em escala regional e global (Leisner, 2020). Entre os desastres ambientais, a seca possui grande relevância, sendo responsável pela queda de produção, déficit hídrico e em casos extremos, a morte de pessoas (Santos \& Aquino, 2017).

Eventos de secas são mais recorrentes nas regiões áridas e semiáridas, que ocupam boa parte das áreas agricultáveis em torno do globo. Na região semiárida do Brasil, a cada 100 anos ocorrem cerca de 18 a 20 anos de seca, sendo o século XX o mais árido até então, com 27 anos de estiagem (Marengo, 2008). A ocorrência de secas é um fator de risco para segurança hídrica, e principalmente para a agricultura de subsistência nessas regiões, provocando perdas econômicas, que resultam em pobreza e êxodo rural (Amaral et al., 2019; Andrade \& Marques, 2017; Marengo et al., 2018; Tabarelli et al., 2018).

Na pecuária, as alterações climáticas afetam os recursos alimentares, hídricos e interferem na saúde e no conforto dos animais, o que reflete na redução da oferta da proteína animal para alimentação humana, que em nível mundial é responsável por 31\% dos alimentos consumidos diariamente (Godde et al., 2021). Logo, a contribuição da pecuária na segurança alimentar será atingida de forma significativa pelo processo de mudanças climáticas, em magnitudes ainda desconhecidas.

Outro problema recorrente é a desertificação, que consiste no processo de degradação e perda gradativa da capacidade produtiva do solo, decorrente das atividades antropogênicas e das mudanças do clima (Accioly, 2010). Entre as ações antrópicas que contribuem para a desertificação, destacam-se o desmatamento, pastejo intensivo e manejo inadequado da irrigação com água salina. A desertificação ainda está associada a ocorrência de secas prolongadas e a variabilidade do regime pluviométrico, que limita o crescimento da cobertura vegetal, expondo os solos aos intemperes naturais (Santos \& Aquino, 2017).

O nível de susceptibilidade das áreas a desertificação é obtido a partir do Índice de Aridez (Tabela 1), obtido a partir da relação entre a precipitação média anual e a evapotranspiração potencial anual, conforme Thornthwaite, (1948) e aperfeiçoado por Penman-Monteith, (1953). 
Tabela 1. Nível de susceptibilidade a desertificação de acordo com o Índice de Aridez.

\begin{tabular}{cc}
\hline Nível de susceptibilidade à desertificação & Índice de Aridez (IA) \\
\hline Muito alta & 0,05 a 0,20 \\
Alta & $0,20<$ IA $<0,50$ \\
Moderado & $0,51<$ IA $<0,65$ \\
\hline
\end{tabular}

Fonte: Matallo Junior (2003).

No mundo, mais de um bilhão de pessoas são afetadas pela desertificação, gerando prejuízos na faixa US\$ 42 bilhões por ano, enquanto medidas de prevenção custariam entre 10 e 20 bilhões de dólares ano-1 (Tavares et al., 2019). Tais medidas de prevenção podem ser obtidas por meio de cultivos sustentáveis e resilientes, que promovam a cobertura e ocupação do solo, além de aumentar a oferta de forragem para alimentação dos animais em ambiente Semiárido.

\subsection{Produção agropecuária em ambiente Semiárido}

As regiões secas são mais vulneráveis às alterações do clima, quando comparadas as demais regiões, isso se deve aos aspectos naturais como altas temperaturas do ar e elevado déficit hídrico durante grande parte do ano (Carvalho et al., 2015; Mata \& Resende, 2018; Vieira et al., 2020). No mundo, as regiões áridas e semiáridas ocupam cerca de 55\% do território (Hussain et al., 2018). No Brasil, o Semiárido está localizado na região Nordeste do país, com 1.128 .697 km² de extensão (Figura 1), comportando 1.262 municípios e população de 27.870.242 habitantes, sendo a região semiárida mais populosa do mundo (Sudene, 2017).

Figura 1. Nova delimitação da região semiárida brasileira.

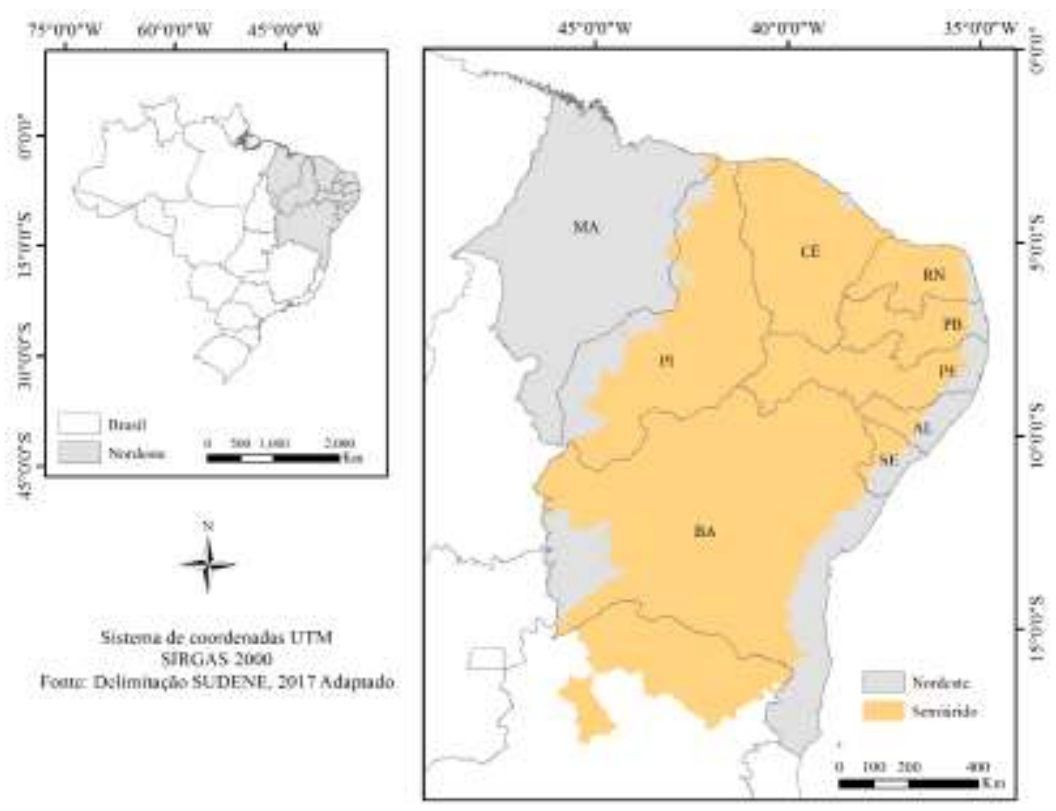

Fonte: Adaptado Sudene (2017).

Essa região é caracterizada por apresentar baixos índices pluviométricos $\left(\leq 800 \mathrm{~mm}^{2} \mathrm{no}^{-1}\right)$, temperatura média do ar entre 23 e $27{ }^{\circ} \mathrm{C}$, demanda atmosférica de $2.000 \mathrm{~mm}^{2} \mathrm{ano}^{-1}$, umidade relativa média do ar em torno de $50 \%$, índice de Aridez de Thornthwaite igual ou inferior a 0,50 e percentual de déficit hídrico anual igual ou superior a 60\% (Moura et al., 2007; Sudene, 2017). Devido a essas características, o processo de mudanças climáticas é mais acentuado. Nos últimos 50 anos a temperatura no Brasil aumentou $0,7^{\circ} \mathrm{C}$, enquanto no Nordeste, esse aumento foi de 1,5 a $2{ }^{\circ} \mathrm{C}$ em apenas 41 anos (Marengo, 2008). Até o 
final do século, a temperatura nessa região deve aumentar entre 2 e $4{ }^{\circ} \mathrm{C}$, além de redução nas chuvas de aproximadamente 15 a 20\%, sob cenário climático mais pessimista (Ferreira, 2017; Marengo, 2008).

A agricultura da região é composta em grande parte pela agricultura familiar, com baixo uso de tecnologia na produção, tornando os sistemas de produção vulneráveis, uma vez que são dependentes das condições climáticas que quase sempre são desfavoráveis. Dessa forma, a maioria dos cultivos são realizados apenas durante o período chuvoso, podendo ainda, ocorrer eventos de veranicos, i. e., período seco dentro da quadra chuvosa, que dependendo da intensidade e duração, provoca danos na lavoura (Carvalho, 2020). A pecuária extensiva é mais comum na região, onde a vegetação nativa é a fonte de alimento para os animais. Entretanto, a Caatinga é submetida à sistemas de manejo inadequado, apresentando como consequência, baixa capacidade de suporte forrageiro, oferta de forragem apenas no período chuvoso e degradação do ecossistema (Bueno \& Rocha, 2018).

\subsection{Adoção de práticas resilientes}

O Painel Intergovernamental sobre Mudanças Climáticas (IPCC) define resiliência agrícola como a capacidade de um sistema e seus componentes de antecipar, adaptar-se ou se recuperar de um evento perigoso de maneira oportuna e eficiente (Field et al., 2012). A resiliência agrícola é um fator importante na manutenção dos sistemas agrícolas, e para enfrentar desafios futuros, como o abastecimento de alimentos para uma população em crescimento, adversidades climáticas, fornecimento de matéria prima para indústria e instabilidade econômica (Chaudhary et al., 2018).

Com isso, sistemas de cultivos resilientes são recomendados para promover o desenvolvimento sustentável, econômico e reduzir a vulnerabilidade do setor agrícola às adversidades climáticas em ambiente semiárido (Douxchamps et al., 2017; Michler et al., 2019; Szymczak et al., 2020; Williams et al., 2020). Os cultivos resilientes são compostos por vários métodos, como a consorciação entre as culturas, escolhas de espécies adaptadas às condições edafoclimáticas da região, densidade de plantio adequada, correção nutricional e manejo do solo, controle de pragas e doenças, uso da irrigação complementar, e uso de cobertura morta, viva ou sintética do solo (Sentelhas \& Monteiro, 2009).

Ensaio experimentais tem demostrado resultados positivos quando aplicado práticas de resiliência agrícola em ambiente semiárido. Trabalhando com uso de cobertura morta, Souza et al. (2021) observaram melhorias em características morfológica e de crescimento no cultivo de palma forrageira em ambiente semiárido. Em sistema de consorcio palma-sorgo, Jardim et al. (2021) obtiveram incremento de $47 \%$ na produção de matéria fresca e 3,5 vezes a mais na produção de matéria seca, quando comparado ao cultivo exclusivo de palma forrageira. Estudando a densidade de plantio em cultivo de palma forrageira irrigada com efluente de esgoto doméstico, Lemos et al. (2021) alcançaram produtividade superiores a $900 \mathrm{t} \mathrm{ha} \mathrm{ano}^{-1}$. Carvalho et al. (2017) afirmam que com a quantidade da precipitação pluvial no Semiárido brasileiro é possível e recomendável a adoção de práticas de resiliência agrícola.

\subsubsection{Palma forrageira: utilização de diferentes genótipos}

A variabilidade sazonal da precipitação pluvial, em escala inter e intra-anual, dificulta a produção de forragem em ambiente semiárido (Dubeux Júnior et al., 2010). Nesse sentido, o incremento produtivo de uma cultura em uma determinada região provém da capacidade de adaptação ao ambiente e do manejo empregado, que facilita a conversão da água, radiação e nutrientes em fotoassimiladados, com ganhos de produtividade (Firincioğlu et al., 2010). Quanto ao manejo, caraterísticas como tolerância a níveis salinos e adensamento são desejáveis. Na região semiárida brasileira grande parte das propriedades rurais são de pequeno porte, e as águas dos poços apresentam elevados teores de sais (Guanziroli et al., 2012; Pimentel \& Sharqawy, 2020). A escolha de espécies forrageiras tolerante ao estresse hídrico e com alta eficácia no uso da água é essencial para manter a produção de forragem estável durante todo o ano. 
Originária do México, a palma forrageira foi introduzida no Brasil em meados do século XIX, com o intuito de produzir o corante carmim a partir da cochonilha Dactylopius coccus, mas a atividade não obteve sucesso, então descobriu-se que a palma possuía potencial forrageiro e, assim, foi destinada para alimentação animal e difundida pela região semiárida. O insucesso na produção do corante se deu pelo fato do equívoco na introdução da cochonilha, foi adquirida a cochonilha do Carmim (Dactylopius opuntiae) que é a principal praga da cultura, e não é eficiente para a produção do corante. Essa praga dizimou os palmais do Nordeste brasileiro (Lima et al., 2017; Vasconcelos et al., 2009).

Para recuperar os palmais, foram selecionados clones de palma forrageira do gênero Opuntia e Nopalea, resistentes a cochonilha do carmim. Os clones de maior destaque são a Orelha de Elefante Mexicana (OEM, Opuntia stricta (Haw.) Haw.), Miúda (MIU, Nopalea cochenillifera (L.) Salm-Dyck) e IPA Sertânia (Nopalea cochenillifera (L.) Salm-Dyck), entretanto, outros clones possuem potencial, como a Orelha de Elefante Africana (OEA, Opuntia undulata Griffiths), V19 (Opuntia larreri) e F8 (Opuntia atropes) (Figura 2) (Vasconcelos et al., 2009).

Figura 2. Genótipos de palma forrageira cultivados no município Serra Talhada - PE, Brasil. Orelha de Elefante Mexicana (A), Orelha de Elefante Africana (B), V19 (C), F8 (D), Miúda (E) e IPA Sertânia (F).

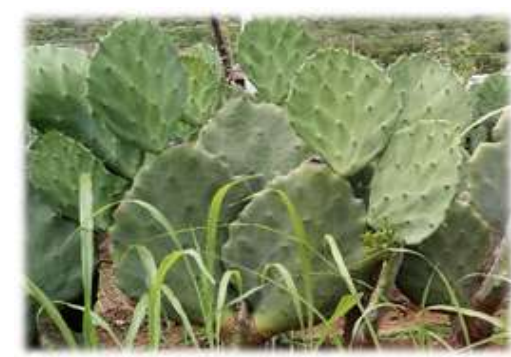

(A)

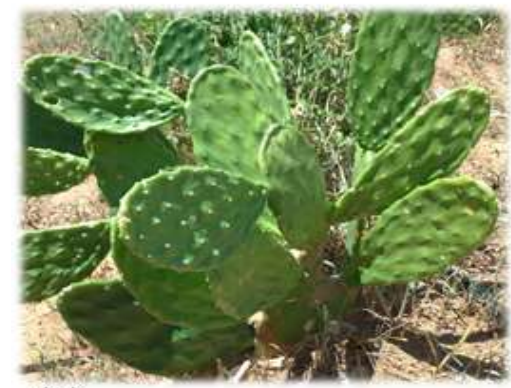

(D)

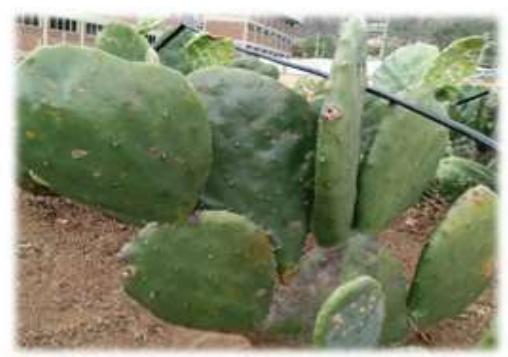

(B)

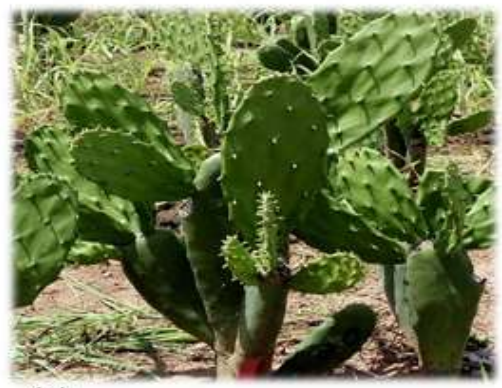

(E)

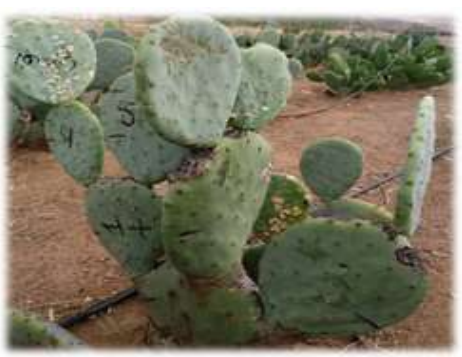

(C)

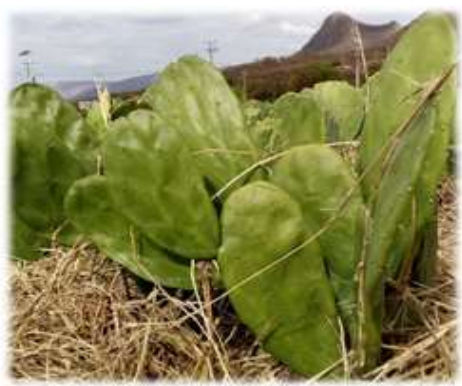

(F)

Fonte: Autores.

A palma forrageira apresenta o metabolismo ácido das crassuláceas (MAC), características morfológicas (cladódios, película espeça) e fisiológicas (abertura dos estômatos durante a noite), que permite uma melhor eficiência no uso da água, nutrientes, tolerância ao estresse hídrico e altas temperaturas, com boa produção de fitomassa. A abertura estomática para captura do $\mathrm{CO}_{2}$ atmosférico no período noturno, reduz as perdas de água por transpiração. $\mathrm{O} \mathrm{CO}_{2}$ capturado é convertido em malato e armazenado nos vacúolos das células na forma de ácido málico. Durando o dia, o malato é transportado para os cloroplastos, descarboxilado pela enzima NADP-málica, ao passo que o $\mathrm{CO}_{2}$ é liberado e a enzima Rubisco pode atuar da mesma forma que no metabolismo $\mathrm{C}_{3}$, mas com os estômatos fechados, resultando em máxima eficiência no uso da água (Figura 3) (Nunes, 2011; Scalisi et al., 2016; Souza Filho et al., 2016). 
Figura 3. Ilustração simplificada do funcionamento do Metabolismo Ácido das Crassuláceas (MAC).

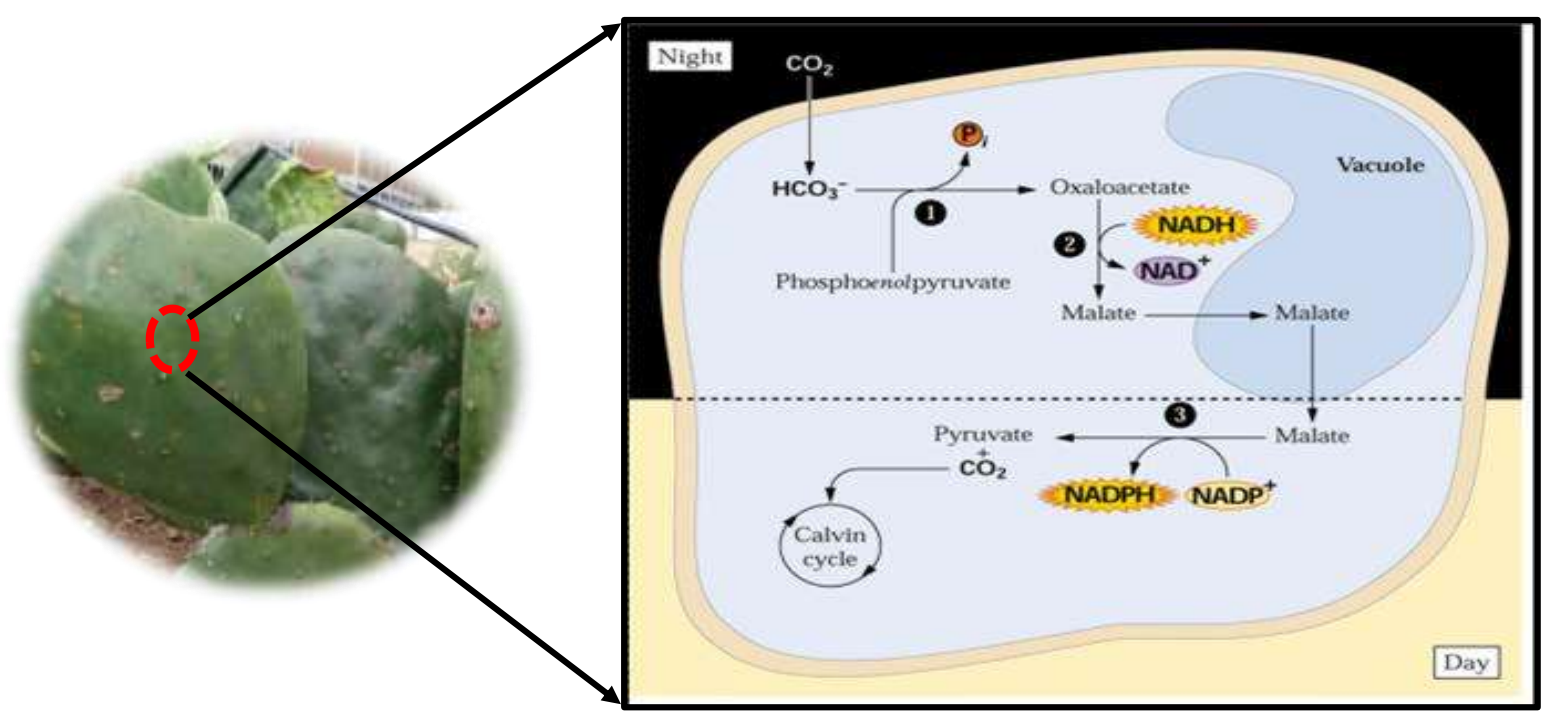

Fonte: Adaptado de Buchanan (2000).

Devido ao metabolismo fotossintético MAC, a palma forrageira apresenta eficiência no uso da água 11x maior, com relação a espécies com metabolismo $\mathrm{C}_{3}$, produzindo quatro vezes mais matéria seca por milímetro de água aplicada (Gusha et al., 2015). Pereira; Cordery; Iacovides. (2012) relataram que as plantas MAC consomem em média 50 kg de água para produzir $1 \mathrm{~kg}$ de matéria seca, enquanto plantas com metabolismo $\mathrm{C}_{3}$ e $\mathrm{C}_{4}$ consomem em média 1000 e $500 \mathrm{~kg}$ de água, respectivamente, para produzir a mesma quantidade de matéria seca. Com relação a perda de água durante as trocas gasosas, as plantas MAC perdem de 50 a $100 \mathrm{~g}$ de água por grama de $\mathrm{CO}_{2}$ obtido, enquanto plantas $\mathrm{C}_{3}$ e $\mathrm{C}_{4}$ chegam a perder $300 \mathrm{~g}$ e $500 \mathrm{~g}$ de água, respectivamente (Taiz \& Zeiger, 2013).

Além disso, no caso da palma forrageira, pequenas precipitações podem ser aproveitadas por essa espécie, isso graças ao sistema radicular superficial distribuído de forma horizontal no solo e o grande potencial osmótico das raízes (Borland et al., 2015; Edvan et al., 2013; Snyman, 2006). Além disso, a palma forrageira apresenta elevada capacidade de armazenamento de água no tecido esponjoso presente nos cladódios (Scalisi et al., 2016). Sendo assim, essa cultura apresenta vantagens produtivas em ambiente semiárido.

A palma forrageira é uma excelente fonte nutricional para os ruminantes, possuindo alto teor de carboidratos solúveis, alta digestibilidade e grande produtividade de matéria fresca (Cardoso et al., 2019; Moura et al., 2020; Santos et al., 2020). Além disso, pode reduzir o consumo de água pelos animais, já que possui $90 \%$ de água em sua constituição, podendo ainda, ser utilizada na recuperação de áreas degradadas (Cheikh Rouhou et al., 2018).

Vários genótipos de palma forrageira estão distribuídos pela região semiárida brasileira (Freire, 2012; Lopes, 2010). As divergências genéticas entre os genótipos revelam graus variados de tolerâncias aos estresses abióticos observados em ambiente Semiárido (Jardim et al. 2021b). Diversos estudo tem avaliado o crescimento e a produtividade de clones de palma forrageira em regiões e condições de manejo distintas (Cavalcante et al., 2014; Rocha et al., 2017; Silva et al., 2015). O cultivo de genótipos mais adaptados a região resulta em ganhos de produtividade.

Estudando seis clones de palma forrageira, Jardim et al. (2021b) observaram os maiores rendimentos de matéria seca para o clone Orelha de Elefante Mexicana, sendo cerca de 34\% mais produtivo quando comparados aos clones V19, IPA Sertânia ou Miúda, e 54\% que os clones F8 e Orelha de Elefante Africana. Para a concentração de carboidratos, os mesmos autores encontraram maior acúmulo no clone OEA, correspondente a 59\% a mais que os clones OEM e F8, que apresentaram 
os menores valores. Para a concentração de potássio $\left(\mathrm{K}^{+}\right)$, observaram os maiores valores no clone OEM, sendo 66\% superior em comparação aos demais clones. Esses autores afirmam que a eficiência na captação de $\mathrm{K}^{+}$reflete uma maior capacidade de reduzir a concentração de sódio $\left(\mathrm{Na}^{+}\right)$nos cladódios, o que torna o clone OEM mais tolerante aos efeitos tóxicos dos sais. No mesmo trabalho, foi evidenciado que os clones F8, V19 e Miúda não são eficientes no uso de elétrons no processo de fixação de carbono, ocasionando danos ao fotossistema II. Essa baixa eficiência promove alta dissipação de energia, e indica a baixa capacidade de adaptação desses clones às condições estressantes do clima Semiárido (Jardim et al., 2021b).

\subsubsection{Irrigação suplementar como estratégia mitigatória às adversidades climáticas do semiárido}

Por mais que a palma forrageira apresente tolerância ao déficit hídrico, vários estudos têm demostrado incremento na produtividade, com uso da irrigação (Dubeux et al., 2015; Lemos et al., 2021; Pereira et al., 2015; Queiroz et al., 2015). Sob condições de déficit hídrico a palma forrageira apresenta murcha e baixo desempenho produtivo (Lemos et al., 2021). Nesse aspecto, a irrigação é fundamental para atender a necessidade de água da cultura e diminuir os riscos de perdas na produção.

Em condições irrigadas, a palma forrageira (Opuntia spp. e Nopalea spp.) pode produzir entre 50 e 60 vezes mais forragem por hectare em comparação a Caatinga (Dubeux et al., 2015). Queiroz et al. 2015 estudando o efeito da aplicação de diferentes lâminas de irrigação para o clone Orelha de Elefante Mexicana, determinaram que as lâminas de irrigação na faixa de 1048 e 1096 mm para o ciclo de 12 meses, promovem os melhores resultados. Araújo Júnior et al. (2021) avaliando os benéficos econômicos do uso da irrigação na palma forrageira, constataram que no primeiro ciclo de cultivo (18 meses) com os clones OEM e Miúda, os custos com a montagem do sistema e irrigação e plantio da cultura são pagos e é obtido retorno econômico.

Outros estudos apontam que a palma forrageira requer entre 50-75 $\mathrm{m}^{3}$ de água ha ${ }^{-1}$ ao mês para se obter alto rendimento de biomassa (Lemos et al., 2021). Santos et al. (2017) estudando as lâminas e frequência de irrigação, concluíram que a lâmina correspondente a 33\% da Evapotranspiração de referência (ETo) aplicada na frequência de três dias, apresentou os melhores resultados. Sob condições irrigadas, a palma forrageira pode apresentar rendimentos de matéria fresca na faixa de $600 \mathrm{t} \mathrm{ha}^{-1}$, e em condições de sequeiro a produtividade média varia entre 120-160 t ha ${ }^{-1}$ (Lima et al., 2015; Silva et al., 2015).

Experimentos mais detalhadas foram realizados por Queiroz et al. (2015), para determinar a exigência hídrica da palma forrageira, como o Kc - coeficiente de cultivo, que de acordo com os autores é 0,52. O sistema de irrigação mais indicado para essa cultura e para as condições climáticas do semiárido é a irrigação por gotejamento, que diminui as perdas de água por evaporação ou percolação e aumenta a eficiência na aplicação da água (Santos et al., 2020). No entanto, apenas 2-3\% da região semiárida é apta para irrigação, devido a condições do solo e limitações de água (Dubeux Júnior et al., 2010). Dessa forma, é necessário buscar fontes alternativas de águas para irrigação na cultura da palma forrageira.

\subsubsection{Agricultura biossalina}

Agricultura biossalina é o cultivo de plantas convencionais tolerantes aos sais ou de plantas halófitas, irrigadas com água salina de diversas fontes e teores salinos (Khorsandi et al., 2020). Para evitar o acúmulo de sais no solo, a água deve ser manejada em quantidades recomendadas, e os solos devem apresentar alta permeabilidade. Em regiões com água de melhor qualidade, é recomendado a aplicação da lâmina de lixiviação com o intuito de carrear o excesso de sais, caso os níveis de precipitação pluvial não sejam suficientes para essa prática (Costa et al., 2013; Lima. et al., 2007).

No mundo, cerca de 30 milhões de hectares são prejudicados pelo manejo inadequado da irrigação com águas de elevados teores salinos e, desses, ao menos 0,25 e 0,5 milhões de hectares são perdidos anualmente pela salinização dos solos (FAO, 2002). No Brasil, grande parte dos solos da região Nordeste são formados sob o cristalino, composto por rochas ígneas 
e metamórficas, que em contato com a água liberam sais solúveis de sua composição (Porto et al., 2019). Tal processo, resulta na salinização das águas subterrâneas, que por sua vez, são utilizadas na produção de alimentos.

A quantidade de sais presente na água é avaliada pela condutividade elétrica (CE), que de acordo com Richards (1954) a classificação é dividida em águas com salinidade baixa (CE entre 0,10 e $0,25 \mathrm{dS} \mathrm{m}^{-1}$ ), salinidade média (CE entre 0,25 e $0,75 \mathrm{dS} \mathrm{m}^{-1}$ ), salinidade alta (CE entre 0,75 e $2,25 \mathrm{dS} \mathrm{m}^{-1}$ ) e salinidade muito alta (CE superior a $2,25 \mathrm{dS} \mathrm{m}^{-1}$ a $25^{\circ} \mathrm{C}$ ). Estudando a qualidade das águas superficiais e subterrâneas em 33 municípios da zona semiárida do estado de Pernambuco, Nunes Filho et al. (2000) mostraram que 38\% das águas subterrâneas e 45\% das águas superficiais apresentaram salinidade média, enquanto $28 \%$ e $30 \%$, na mesma sequência, apresentaram salinidade alta. Nesse sentido, águas com elevados teores salinos podem ser manejadas de forma sustentável em áreas implantadas com espécies tolerantes aos sais (Masters et al., 2007).

Estudando o mecanismo de tolerância das plantas a salinidade, Munns; Tester, (2008) afirmam que a salinidade do extrato de saturação do solo na faixa de 0,76 a $2,0 \mathrm{dS} \mathrm{m}^{-1}$ não afeta o crescimento da maioria das forragens, enquanto valores na faixa de 2,0 a 3,5 $\mathrm{dS} \mathrm{m}^{-1}$ pode interferir no desenvolvimento das plantas, inclusive da palma forrageira. Para a Organização das Nações Unidas para Alimentação e Agricultura - FAO (2005), o solo é considerado salino quando a CE for superior a 4 dS $\mathrm{m}^{-1}$.

Aplicando-se água com condutividade elétrica de $3,6 \mathrm{dS} \mathrm{m}^{-1}$ no cultivo de 20 genótipos de palma forrageira, Freire (2012) obteve as maiores produtividades com os clones Orelha de Elefante Mexicana e Africana, enquanto a menor produtividade foi observada no clone F-8. Lima et al. (2018) não observaram redução na produtividade do clone Orelha de Elefante Mexicana em virtude da irrigação com água de salinidade igual a 1,6 dS m $\mathrm{m}^{-1}$. Desse modo, a utilização de irrigação com água salina pode ser uma alternativa para o cultivo de clones de palma forrageira, todavia, mais estudos precisam ser realizados para avaliar a eficiência de diferentes cultivares de palma forrageira à essas condições.

\section{Considerações Finais}

As mudanças climáticas devem afetar a produção de forragem em todo o globo, com maior severidade nas regiões Semiáridas, reduzindo a oferta de animais para o mercado e, dessa forma, aumentando a insegurança na alimentação humana. Para contornar esse problema, a literatura recomenda a utilização de espécies forrageiras que sejam tolerantes as condições adversas do clima. Nesse sentido, na região Semiárida brasileira, a cultura de maior destaque é a palma forrageira, em especial a cultivar Orelha de Elefante Mexicana (Opuntia stricta (Haw.) Haw.), que tem se sobressaído, em termos de resistência e produção, em relação a outros clones. Associado a escolha da espécie de planta forrageira adequada, a implantação de práticas de resiliência agrícola, como o uso da irrigação complementar, pode incrementar a produtividade da cultura, aumentando a oferta de alimento para os animais e reduzindo os riscos associados à variabilidade da chuva. A agricultura biossalina em cultivos de palma forrageira tem se mostrado uma prática promissora, contudo, é necessário o uso adequado da água de irrigação, tornando os sistemas de produção dessa cactácea mais sustentáveis. Logo, a garantia de um melhor aporte forrageiro ao longo do ano é facilitada.

Informações sobre o crescimento, por meio de índices morfofisiológicos e avaliação fenológica, e em relação a produtividade dos genótipos Orelha de Elefante Africana (Opuntia undulata Griffiths), V19 (Opuntia larreri) e F8 (Opuntia atropes) submetidos à distintas condições de cultivo (e.g., alta densidade populacional de plantas; sequeiro; irrigado com água residuária e/ou salina; uso de cobertura morta sobre o solo; orientação de plantio; orientação de cultivo; frequências e intensidades de corte; consorciação de culturas, entre outros) ainda são escassas. Portanto, é encorajado que mais estudos sejam realizados para avaliar as respostas adaptativas desses clones de palma forrageira ao manejo empregado, de forma a 
recomendar a inserção de outros genótipos de palma forrageira e, ainda, incrementar os rendimentos da cultura nos sistemas de produção do Semiárido brasileiro.

\section{Agradecimentos}

Os autores agradecem ao Conselho Nacional de Desenvolvimento Científico e Tecnológico - CNPq e a Coordenação de Aperfeiçoamento de Pessoal de Nível Superior - CAPES, pelo apoio financeiro. Os autores também agradecem ao Grupo de Agrometeorologia no Semiárido - GAS, pelo auxílio no desenvolvimento da pesquisa.

\section{Referências}

Amaral, A. P. M. C., Marchezini, V., Lindoso, D. P., Saito, S. M., \& Santos Alvalá, R. C. (2019). Desafios para a consolidação de um sistema de alerta de risco de desastre associado às secas no Brasil. Sustentabilidade em Debate, 10(1), 60-76.

Amorim, P. L., Martuscello, J. A., Filho, J. T. D. A., Cunha, D. D. N. F. V., \& Jank, L. (2015). Morphological and productive characterization of forage cactus varieties. Revista Caatinga, 28(3), 230-238. https://doi.org/10.1590/1983-21252015v28n326rc.

Andrade, C. M., \& Marques, L. D. S. (2017). Semiárido brasileiro: alguns desafios. Diversitas Journal, 2(2), 279. https://doi.org/10.17648/diversitas-journalv2i 2.567 .

Araújo Júnior, G. D. N., Silva, T. G. F., Souza, L. S. B., Souza, M. S., Araújo, G. G. L., Moura, M. S. B., Santos, J. P. A. de S., Jardim, A. M. R. F., Alves, C. P., \& Alves, H. K. M. N. (2021). Productivity, bromatological composition and economic benefits of using irrigation in the forage cactus under regulated deficit irrigation in a semiarid environment. Bragantia, 80, 1-12. https://doi.org/10.1590/1678-4499.20200390.

Barbosa, M. L., Silva, T. G. F., Zolnier, S., e Silva, S. M. S., \& Ferreira, W. P. M. (2018). Environmental variables influencing the expression of morphological characteristics in clones of the forage cactus. Revista Ciencia Agronomica, 49(3), 399-408. https://doi.org/10.5935/1806-6690.20180045.

Borland, A. M., Wullschleger, S. D., Weston, D. J., Hartwell, J., Tuskan, G. A., Yang, X., \& Cushman, J. C. (2015). Climate-resilient agroforestry: Physiological responses to climate change and engineering of crassulacean acid metabolism (CAM) as a mitigation strategy. Plant, Cell and Environment, 38(9), 1833-1849. https://doi.org/10.1111/pce.12479.

Bueno, L. G., \& Rocha, J. E. S. (2018). Conservação, utilização e melhoramento genético de gramíneas forrageiras para o Semiárido brasileiro. Embrapa Caprinos e Ovinos - Documentos (Infoteca-E), 33p.

Cardoso, D. B., Carvalho, F. F. R., Medeiros, G. R., Guim, A., Cabral, A. M. D., Véras, R. M. L., Santos, K. C. dos, Dantas, L. C. N., \& Nascimento, A. G. O. (2019). Levels of inclusion of spineless cactus (Nopalea cochenillifera Salm Dyck) in the diet of lambs. Animal Feed Science and Technology, 247, 23-31. https://doi.org/10.1016/j.anifeedsci.2018.10.016.

Carvalho, A. A. d., Silva, T. G. F. d., Souza, L. S. B. d., Moura, M. S. B. d., Araujo, G. G. L. d., \& Tolêdo, M. P. S. (2017). Soil moisture in forage cactus plantations with improvement practices for their resilience. Revista Brasileira de Engenharia Agrícola e Ambiental, 21(7), 481 487.https://doi.org/10.1590/1807-1929/agriambi.v21n7p481-487.

Carvalho, A. T. F. (2020). Caracterização climática da quadra chuvosa de município do semiárido brasileiro, entre os anos de 2013 a 2017. Geografia Em Atos (Online), 2(17), 04-23. https://doi.org/10.35416/geoatos.v2i17.7116.

Carvalho, A. L. de, Menezes, Rô. S. C., Nóbrega, R. S., Pinto, A. S., Ometto, J. P. H. B., von Randow, C., \& Giarolla, A. (20 15). Impact of climate changes on potential sugarcane yield in Pernambuco, northeastern region of Brazil. Renewable Energy, 78, 26-34. https://doi.org/10.1016/j.renene.2014.12.023.

Cavalcante, L. A. D., Santos, G. R. A., Silva, L. M., Fagundes, J. L., \& Silva, M. A. (2014). Respostas de genótipos de palma forrageira a diferentes densidades de cultivo. Pesquisa Agropecuária Tropical, 44(4), 424-433. https://doi.org/10.1590/s1983-40632014000400010.

Chaudhary, A., Gustafson, D., \& Mathys, A. (2018). Multi-indicator sustainability assessment of global food systems. Nature Communications, 9, 848. https://doi.org/10.3929/ethz-b-000244685.

Cheikh Rouhou, M., Abdelmoumen, S., Thomas, S., Attia, H., \& Ghorbel, D. (2018). Use of green chemistry methods in the extraction of dietary fibers from cactus rackets (Opuntia ficus indica): Structural and microstructural studies. International Journal of Biological Macromolecules, 116, 901https://doi.org/10.1016/j.ijbiomac.2018.05.090.

Chen, Y., Li, Z., Fan, Y., Wang, H., \& Deng, H. (2015). Progress and prospects of climate change impacts on hydrology in the arid region of northwest China. Environmental Research, 139, 11-19. https://doi.org/10.1016/j.envres.2014.12.029.

Costa, M. E. da, Morais, F. A., Souza, W. C. M., Gurgel, M. T., \& Oliveira, F. H. T. de. (2013). Estratégias de irrigação com água salina na mamoneira. Revista Ciência Agronômica, 44(1), 34-43. https://doi.org/10.1590/s1806-66902013000100005.

Douxchamps, S., Debevec, L., Giordano, M., \& Barron, J. (2017). Monitoring and evaluation of climate resilience for agricultural development - A review of currently available tools. World Development Perspectives, 5, 10-23. https://doi.org/10.1016/j.wdp.2017.02.001.

Dubeux, J. C. B., Santosa, M. V. F., Melloa, A. C. L., Vieira Cunha, M., Ferreira, M. D. A., Santos, D. C., Lira, M. D. A., \& Silva, M. D. C. (2015). Forage potential of cacti on drylands. Acta Horticulturae, 1067, 181-186. https://doi.org/10.17660/actahortic.2015.1067.24. 
Dubeux Júnior, J. C. B., Araújo Filho, J. T., Santos, M. V. F., Lira, M. A., Santos, D. C., \& Pessoa, R. A. S. (2010). Adubação mineral no crescimento e composição mineral da palma forrageira -Clone IPA-201. Revista Brasileira de Ciências Agrária, 5(1), 129-135. https://doi.org/10.5039/agraria.v5i1a591.

Edvan, R. L., Fernandes, P. D., Socorro, M., Carneiro, D. S., Silva, J., Andrade, A. P., Teixeira, L., \& Filho, S. (2013). Acúmulo de biomassa e crescimento radicular da palma forrageira em diferentes épocas de colheita. Revista Acadêmica de Ciências Agrárias e Ambientais, 11(4), 373-381.

FAO. (2002). Crops and Drops: Making the Best Use of Water for Agriculture. Aging, 7(11), 956-963.

FAO. (2016). The State of Food Security and Nutrition in the World 2016. Agrovoc, 320 p.

Ferreira, L. C. (2017). O Desafio das mudanças climáticas: os casos Brasil e China. Paco Editorial, 312.

Field, C. B. (2012). Gerenciando os riscos de eventos extremos e desastres para promover a adaptação às mudanças climáticas: relatório especial do painel intergovernamental sobre mudanças climáticas. Cambridge University Press.

Firincioğlu, H. K., Ünal, S., Erbektaş, E., \& Doğruyol, L. (2010). Relationships between seed yield and yield components in common vetch (Vicia sativa ssp. sativa) populations sown in spring and autumn in central Turkey. Field Crops Research, 116(1-2), 30-37. https://doi.org/10.1016/j.fcr.2009.11.005.

Freire, J. L. (2012). Avaliação de clones de palma forrageira (opuntia e nopalea) sob irrigação e salinidade. Tese (Doutorado em Zootecnia) Universidade Federal Rural de Pernambuco, Recife, BR-PE.

Godde, C. M., Mason-D’Croz, D., Mayberry, D. E., Thornton, P. K., \& Herrero, M. (2021). Impacts of climate change on the livestock food supply chain; a review of the evidence. Global Food Security, 28. https://doi.org/10.1016/j.gfs.2020.100488.

Guanziroli, C. E., Buainain, A. M., \& Di Sabbato, A. (2012). Dez Anos de Evolução da Agricultura Familiar no Brasil: (1996 e 2006). Revista de Economia e Sociologia Rural, 50(2), 351-370. https://doi.org/10.1590/S0103-20032012000200009.

Gusha, J., Halimani, T. E., Katsande, S., \& Zvinorova, P. I. (2015). The effect of Opuntia ficus indica and forage legumes-based diets on goat productivity in smallholder sector in Zimbabwe. Small Ruminant Research, 125, 21-25. https://doi.org/10.1016/j.smallrumres.2015.02.018.

Hussain, J., Khaliq, T., Ahmad, A., Akhter, J., \& Asseng, S. (2018). Wheat Responses to Climate Change and Its Adaptations: A Focus on Arid and Semi-arid Environment. International Journal of Environmental Research, 12(1), 117-126. https://doi.org/10.1007/s41742-018-00742.

Jardim, A. M. R. F., Santos, H. R. B., Alves, H. K. M. N., Silva, S. L. F., Souza, L. S. B., Araújo Júnior, G. N., Souza, M. S., Araújo, G. G. L., Souza, C. A. A. de, \& Silva, T. G. F. (2021). Genotypic differences relative photochemical activity, inorganic and organic solutes and yield performance in clones of the forage cactus under semi-arid environment. Plant Physiology and Biochemistry, 162, 421-430. https://doi.org/10.1016/j.plaphy.2021.03.011.

Jardim, A. M. R. F., Silva, T. G. F., Souza, L. S. B. de, Araújo Júnior, G. N., Alves, H. K. M. N., Souza, M. de S., Araújo, G. G. L., \& Moura, M. S. B. (2021). Intercropping forage cactus and sorghum in a semi-arid environment improves biological efficiency and competitive ability through interspecific complementarity. Journal of Arid Environments, 188. https://doi.org/10.1016/j.jaridenv.2021.104464.

Khorsandi, F., Siadati, S. M. H., \& Rastegary, J. (2020). Haloengineering as a vital component of sustainable development in salt-affected ecosystems. Environmental Development, 35. https://doi.org/10.1016/j.envdev.2020.100545.

Leisner, C. P. (2020). Review: Climate change impacts on food security- focus on perennial cropping systems and nutritional value. Plant Science, 293. https://doi.org/10.1016/j.plantsci.2020.110412.

Lemos, M., Ferreira-Neto, M., Fernandes, C. S., Bezerra, Y. L., Dias, N. S., Medeiros, J. F., Brito, R. F., \& Sá, F. V S. (2021). The effect of domestic sewage effluent and planting density on growth and yield of prickly pear cactus in the semiarid region of Brazil. Journal of Arid Environments, 185. https://doi.org/10.1016/j.jaridenv.2020.104372.

Lima, G. F. C., Silva, J. G. M., Dantas, F. D. G., Guedes, F. X., Rêgo, M. M. T., Aguiar, E. M., \& Lôbo, R. N. B. (2015). Effect of different cutting intensities on morphological characteristics and productivity of irrigated Nopalea forage cactus. Acta Horticulturae, 1067, 253-258. https://doi.org/10.17660/ActaHortic.2015.1067.35.

Lima, L. R., Silva, T. G. F., Pereira, P. C., Morais, J. E. F., \& Assis, M. C. S. (2018). Productive-economic benefit of forage cactus sorghum intercropping systems irrigated with saline water. Revista Caatinga, 31(1), 191-201. https://doi.org/10.1590/1983 21252018v31n122rc.

Lima, M., Silva, D., Ferreira, W., Silva, L., \& Paranhos, B. (2017). Predadores associados a Dactylopius Opuntiae (Hemiptera: Dactylopiidae) em palma forrageira no estado de Pernambuco, Brasil. Revista Chilena de Entomologia, 36(0).

Loladze, I. (2014). Hidden shift of the ionome of plants exposed to elevated $\mathrm{CO}_{2}$ depletes minerals at the base of human nutrition. ELife, 2014(3). https://doi.org/10.7554/eLife.02245.

Lopes, E. B. (2010). Seleção de genótipos de palma forrageira (Opuntia spp. e Nopalea spp.) resistentes à cochonilha-do-carmim (Dactylopius opuntiae Cockerell, 1929) na Paraíba, Brasil. Engenharia Ambiental-Pesquisa e Tecnologia, 7(1), 204-2015.

Marengo, J. A. O., Alves, L. M., Alvala, R. C. S., Cunha, A. P., Brito, S., \& Moraes, O. L. L. (2018). Climatic characteristics of the 2010-2016 drought in the semiarid northeast Brazil region. Anais da Academia Brasileira de Ciencias, 90(2), 1973-1985. https://doi.org/10.1590/0001 3765201720170206.

Marengo, J. a. (2008). Vulnerabilidade, impactos e adaptação à mudança do clima no semiárido do Brasil. Parcerias Estratégicas, 13(27), 149 176. http://seer.cgee.org.br/index.php/parcerias_estrategicas/article/view/329.

Masters, D. G., Benes, S. E., \& Norman, H. C. (2007). Biosaline agriculture for forage and livestock production. Agriculture, Ecosystems and Environment, 119(3-4), 234-248. https://doi.org/10.1016/j.agee.2006.08.003. 
Mata, D., \& Resende, G. (2018). Changing the Climate for Banking: The Economic Effects of Credit in a Climate-Vulnerable Area. SSRN Electronic Journal. https://doi.org/10.2139/ssrn.3279027.

Mcgrath, J. M., \& Lobell, D. B. (2013). Reduction of transpiration and altered nutrient allocation contribute to nutrient decline of crops grown in elevated $\mathrm{CO}_{2}$ concentrations. Plant, Cell and Environment, 36(3), 697-705. https://doi.org/10.1111/pce.12007.

Medeiros, A. S., Malta, F. M. S., Santos, T. C., \& Gomes, T. C. A. (2020). Soil carbon losses in conventional farming systems due to land-use change in the Brazilian semi-arid region. Agriculture, Ecosystems and Environment, 287. https://doi.org/10.1016/j.agee.2019.106690.

Michler, J. D., Baylis, K., Arends-Kuenning, M., \& Mazvimavi, K. (2019). Conservation agriculture and climate resilience. Journal of Environmental Economics and Management, 93, 148-169. https://doi.org/10.1016/j.jeem.2018.11.008.

Moraes, G. S. O., Guim, A., Tabosa, J. N., Chagas, J. C. C., Almeida, M. de P., \& Ferreira, M. A. (2019). Cactus [Opuntia stricta (Haw.) Haw] cladodes and corn silage: How do we maximize the performance of lactating dairy cows reared in semiarid regions? Livestock Science, 221, 133-138. https://doi.org/10.1016/j.livsci.2019.01.026.

Moura, M. S. C., Guim, A., Batista, Â. M. V., Maciel, M. V., Cardoso, D. B., Lima Júnior, D. M. de, \& Carvalho, F. F. R. (2020). The inclusion of spineless cactus in the diet of lambs increases fattening of the carcass. Meat Science, 160. https://doi.org/10.1016/j.meatsci.2019.107975.

Moura, M. S. B., Galvincio, J. D., Brito, L. T. L., Souza, L. S. B., Sá, I. I. S., \& Silva, T. G. F. (2007). Potencialidades da Água de Chuva No Semiárido Brasileiro. Embrapa Semiárido. http://ainfo.cnptia.embrapa.br/digital/bitstream/CPATSA/36534/1/OPB1515.pdf.

Munns, R., \& Tester, M. (2008). Mechanisms of salinity tolerance. Annual Rev. Plant Biology, 59, 651-681. https://doi.org/10.1146/annurev.arplant.59.032607.092911.

Myers, S. S., Zanobetti, A., Kloog, I., Huybers, P., Leakey, A. D. B., Bloom A. J., \& Carlisle, E. (2014). Increasing CO 2 threatens human nutrition. Nature, 510(7503), 139-142.

Neupane, D., Mayer, J. A., Niechayev, N. A., Bishop, C. D., \& Cushman, J. C. (2021). Five-year field trial of the biomass productivity and water input response of cactus pear (Opuntia spp.) as a bioenergy feedstock for arid lands. GCB Bioenergy, 13(4), 719-741. https://doi.org/10.1111/gcbb.12805.

Nunes, C. S. (2011). Usos e aplicações da palma forrageira fomo uma grande fonte de economia para o Semiárido nordestino. Revista Verde, 6(1), 58-66.

Nunes Filho, J., Sousa, A. R., Sá, V. A. L. e, \& Lima, B. P. (2000). Relações entre a concentração de íons e a salinidade de águas subterrâneas e superficiais, visando à irrigação, no sertão de Pernambuco. Revista Brasileira de Engenharia Agrícola e Ambiental, 4(2), 189 193. https://doi.org/10.1590/s141543662000000200010

Nunes, J. D. S. L., Silva, T. G. F., Souza, L. S. B., Jardim, A. M. da R. F., Alves, H. K. M. N., Cruz Neto, J. F., Leite, R. M. C., \& Pinheiro, A. G. (2020). Morfogênese da palma forrageira sob modificação do ambiente de crescimento. Agrometeoros, 27(2). https://doi.org/10.31062/agrom.v27i2.26449.

Palit, P., Kudapa, H., Zougmore, R., Kholova, J., Whitbread, A., Sharma, M., \& Varshney, R. K. (2020). An integrated research framework combining genomics, systems biology, physiology, modelling and breeding for legume improvement in response to elevated $\mathrm{CO}_{2}$ under climate change scenario. Current Plant Biology, 22. https://doi.org/10.1016/j.cpb.2020.100149.

Pereira, A. S., Shitsuka, D. M., Parreira, F. J., \& Shitsuka, R. (2018). Metodologia da pesquisa científica. Santa Maria, Rio Grande do Sul, Ed. UAB/NTE/UFSM.

Pereira, L. S., Cordery, I., \& Iacovides, I. (2012). Improved indicators of water use performance and productivity for sustainable water conservation and saving. Agricultural Water Management, 108, 39-51. https://doi.org/10.1016/j.agwat.2011.08.022.

Pereira, P. D. C., Silva, T. G. F., Zolnier, S., Morais, J. E. F., \& Santos, D. C. (2015). Morfogênese da palma forrageira irrigada por gotejamento. Revista Caatinga, 28(3), 184-195. https://doi.org/10.1590/1983-21252015v28n321rc.

Pimentel, G. D. S., \& Sharqawy, M. H. (2020). Techno-economic analysis of low impact solar brackish water desalination system in the Brazilian Semiarid region. Journal of Cleaner Production, 248. https://doi.org/10.1016/j.jclepro.2019.119255.

Porto, E. R., Hermes, L. C., Ferreira, R. S., Veiga, H. P., \& Saia, A. (2019). Agricultura biossalina: desafios e alternativas para o uso de águas salobras e salinas no semiárido brasileiro. Embrapa Meio Ambiente-Documentos (INFOTECA-E), 38p.

Queiroz, M. G., Silva, T. G. F., Zolnier, S., Silva, S. M. S., Lima, L. R., \& Alves, J. O. (2015). Características morfofisiológicas e produtividade da palma forrageira em diferentes lâminas de irrigação. Revista Brasileira de Engenharia Agrícola e Ambiental, 19(10), 931 938. https://doi.org/10.1590/18071929/agriambi.v19n10p931-938.

Queiroz, M. G., Silva, T. G. F., Zolnier, S., Jardim, A. M. R. F., Souza, C. A. A., Araújo Júnior, G. N., Morais, J. E. F., \& Souza, L. S. B. (2020). Spatial and temporal dynamics of soil moisture for surfaces with a change in land use in the semi-arid region of Brazil. Catena, 188 . https://doi.org/10.1016/j.catena.2020.104457.

Richards, L. A. (1954). Diagnosis and improvement of saline and alkali soils (D. Dept. of Agriculture, Washington (ed.); Agriculture).

Rocha, R. S., Voltolini, T. V., \& Gava, C. A. T. (2017). Características produtivas e estruturais de genótipos de palma forrageira irrigada em diferentes intervalos de corte. Archivos de Zootecnia, 66(255), 363-371. https://doi.org/10.21071/az.v66i255.2512.

Santos, F. D. A., \& Aquino, C. M. S. (2017). Panorama da desertificação no nordeste do brasil: características e suscetibilid ades. Revista de Geografia e Interdisciplinaridade, 2(7), 144. https://doi.org/10.18764/2446-6549.v2n7p144-161. 
Santos, M. R., Donato, S. L. R., \& Cotrim Jr, P. R. F. (2020). Irrigação na palma forrageira. Revista Agrotecnologia, $11(1), 9452$.

Santos, M. R., Silva, A. J. P., Fonseca, V. A., Campos, A. R. F., \& Lisboa, M. A. (2017). Irrigação na palma forrageira. Informe Agropecuário, 38(296), 7690 .

Santos, N. S., Pereira, W. S., Melo, R., Lima, K. V., Lima, D. O., \& Almeida, S. (2020). Crescimento da palma forrageira sob estresse salino e diferentes lâminas de irrigação. Revista Craibeiras de Agroecologia, 5, 9452.

Scalisi, A., Morandi, B., Inglese, P., \& Bianco, R. L. (2016). Cladode growth dynamics in Opuntia ficus-indica under drought. Environmental and Experimental Botany, 122, 158-167. https://doi.org/10.1016/j.envexpbot.2015.10.003.

Scheelbeek, P. F. D., Bird, F. A., Tuomisto, H. L., Green, R., Harris, F. B., Joy, E. J. M., Chalabi, Z., Allen, E., Haines, A., \& Dangour, A. D. (2018). Effect of environmental changes on vegetable and legume yields and nutritional quality. Proceedings of the National Academy of Sciences of the United States of America, 115(26), 6804-6809. https://doi.org/10.1073/pnas.1800442115.

Sentelhas, P. C., \& Monteiro, J. E. B. de A. (2009). Agrometeorologia dos cultivos: o fator meteorologico na produção agrícola, Brasília, Brasil: Inmet.

Silva, E. C. B., Lima, J. R. D. S., Antonino, A. C. D., Melo, A. A. S., Souza, E. S., Souza, R. M. S., Silva, V. P., \& Oliveira, C. L. (2020). Efeito da Irrigação Suplementar na Produtividade e Eficiência no uso de água da palma forrageira. Revista Brasileira de Geografia Física, 13(6), 2744. https://doi.org/10.26848/rbgf.v13.6.p2744-2759.

Silva, T. G. F., Primo, J. T. A., Morais, J. E. F., Silva, W. J. D., Souza, C. A. A., \& Silva, M. C. (2015). Crescimento e produtividade de clones de palma forrageira no semiárido e relações com variáveis meteorológicas. Revista Caatinga, 28(2), 10-18.

Snyman, H. A. (2006). Root distribution with changes in distance and depth of two-yers-old cactus pears Opuntia ficus-indica and O. robusta plants. South África Journal of Botany, 72, 434-441.

Souza Filho, P. F., Ribeiro, V. T., Santos, E. S., \& Macedo, G. R. (2016). Simultaneous saccharification and fermentation of cactus pear biomass-evaluation of using different pretreatments. Industrial Crops and Products, 89, 425-433. https://doi.org/10.1016/j.indcrop.2016.05.028.

Souza, M. D. S., Silva, T. G. F., Souza, L. S. B., Alves, H. K. M. N., Leite, R. M. C., Souza, C. A. A., Araújo, G. G. L. D., Campos, F. S., Silva, M. J. D., \& de Souza, P. J. D. O. P. (2021). Growth, phenology and harvesting time of cactus-millet intercropping system under biotic mulching. Archives of Agronomy and Soil Science. https://doi.org/10.1080/03650340.2020.1852553.

Sudene. (2017). Superintendência do desenvolvimento do Nordeste. http://antigo.sudene.gov.br/delimitacao-do-semiarido.

Szymczak, L. S., Carvalho, P. C. F., Lurette, A., Moraes, A., Nunes, P. A. A., Martins, A. P., \& Moulin, C. H. (2020). System diversification and grazing management as resilience-enhancing agricultural practices: The case of crop-livestock integration. Agricultural Systems, 184 . https://doi.org/10.1016/j.agsy.2020.102904.

Tabarelli, M., Leal, I. R., Scarano, F. R., \& Silva, J. M. C. da. (2018). Caatinga: legado, trajetória e desafios rumo à sustentabilidade. Ciência e Cultura, 70(4), 25-29. https://doi.org/10.21800/2317-66602018000400009.

Taiz, L., \& Zeiger, E. (2013). Fisiologia vegetal (Artmed Editora S.A. (ed.)).

Taiz, L., \& Zeiger, E. (2017). Fisiologia e Desenvolvimento vegetal. California, EUA, ed. 6.

Tavares, V. C., Arruda, Í. R. P., \& Silva, D. G. (2019). Desertificação, mudanças climáticas e secas no semiárido brasileiro: uma revisão bibliográfica. Geosul, 34(70), 385-405. https://doi.org/10.5007/2177-5230.2019v34n70p385.

Theurl, M. C., Lauk, C., Kalt, G., Mayer, A., Kaltenegger, K., Morais, T. G., Teixeira, R. F. M., Domingos, T., Winiwarter, W., Erb, K. H., \& Haberl, H. (2020). Food systems in a zero-deforestation world: Dietary change is more important than intensification for climate targets in 2050. Science of the Total Environment, 735. https://doi.org/10.1016/j.scitotenv.2020.139353.

Thornthwaite, C. W. (1948). An Approach toward a Rational Classification of Climate. Geographical Review, 38(1), 55. https://doi.org/10.2307/210739.

Vasconcelos, A. G. V., Lira, M. A., Cavalcanti, V. L. B., Santos, M. V. F., \& Willadino, L. (2009). Seleção de clones de palma forrageira resistentes à Cochonilha do Carmim Dactyplopius sp. (Hemiptera, Dactylopiidae). Revista Brasileira de Zootecnia, 38(1), 827-831. https://doi.org/10.15528/241.

Vieira Filho, J. E. R., \& Fishlow, A. (2017). Agricultura e Indústria no Brasil: inovação e competitividade. Brasília, Brasil: In Ipea. http://www.ipea.gov.br/portal/index.php?option=com_content\&view=article\&id=29768.

Vieira, R. M. S. P., Sestini, M. F., Tomasella, J., Marchezini, V., Pereira, G. R., Barbosa, A. A., Santos, F. C., Rodriguez, D. A., Nascimento, F. R., Santana, M. O., Barreto Campello, F. C., \& Ometto, J. P. H. B. (2020). Characterizing spatio-temporal patterns of social vulnerability to droughts, degradation and desertification in the Brazilian northeast. Environmental and Sustainability Indicators, 5, 100016. https://doi.org/10.1016/j.indic.2019.100016.

Williams, T. G., Guikema, S. D., Brown, D. G., \& Agrawal, A. (2020). Resilience and equity: Quantifying the distributional effects of resilience-enhancing strategies in a smallholder agricultural system. Agricultural Systems, 182. https://doi.org/10.1016/j.agsy.2020.102832. 